\title{
Review of the Third Edition of A Manual of Acarology
}

\author{
BRUCE HALLIDAY
}

CSIRO Entomology, GPO Box 1700, Canberra ACT 2601, Australia. E-mail: Bruce.Halliday@csiro.au

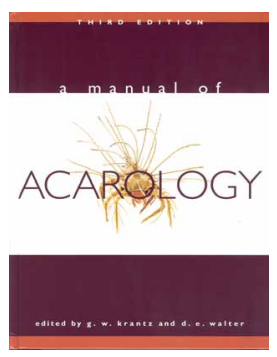

G.W. Krantz and D.E. Walter (editors) A Manual of Acarology, Third Edition. Texas Tech University Press, Lubbock Texas, April 2009 , viii +807 pp.

ISBN 978-0-89672-620-8

Publisher's price USD $\$ 175.00$

Publisher contacts: ttup@ttu.edu, www.ttup.ttu.edu

Every acarology laboratory in the world has a battered and dog-eared copy of the 1978 Second Edition of A Manual of Acarology, by Jerry Krantz. For over 30 years the Manual has been one of the fundamental tools of the trade in the science of acarology, second in importance only to a good microscope. But 30 years is a long life span for any textbook, and the Second Edition of the Manual was definitely starting to show its age. Science has moved on, and has brought with it an explosive increase in our knowledge of mites, both in the sheer numbers of known taxa, and in the development of new technologies that have revealed previously unsuspected levels of complexity and detail. Acarologists have been anxiously awaiting the Third Edition, as a consolidation of the enormous changes that have been going on in acarology at all levels. They will not be disappointed. The number of families of mites has increased from just over 400 in 1978, to an amazing 540 now. There can be very few other animal groups that have grown in family-level diversity at that rate. The growth in the number of taxa covered has been accompanied by significant advances in comparative morphology, genetics, molecular biology, and ecology. These increases in knowledge have been matched by an increase in the size of the Manual, from 509 pages to a hefty 807 pages.

Jerry Krantz wisely decided that a full-scale revision of the Manual was a task beyond the abilities of any single author, so he recruited a team of co-authors, each of whom has contributed chapters in their special field of expertise. The list of authors is a truly impressive one. Most chapters are written or co-written by the two editors, with important chapters by Evert Lindquist, Mark Harvey, Jim Keirans, Ian Smith, David Cook, Roy Norton, Valerie Behan-Pelletier, and Barry OConnor. Most of the authors have taught at the Acarology Summer Program at Ohio State University, which has become an essential part of every acarologist's education. The lecture notes and teaching materials from that course are a rich lode of acarological knowledge, much of which has not been available in consolidated form until now. This new edition of the Manual draws on the wealth of information that has been built up through years of experience there, including feedback from the students, who put preliminary drafts of the identification keys through some rigorous testing.

The first eight chapters include a brief review of the higher-level phylogeny of the mites and their relationship with other arachnid groups, which leaves open the question of whether or not mites are monophyletic. There is an expanded review of external and internal anatomy and the terminology 
used to describe it, and shorter chapters on reproduction and development, embryology, and life cycles. The chapter on Habits and Habitats gives an excellent overview of the enormous diversity of feeding behaviour of mites and of the habitats they occupy. The references cited in this chapter draw heavily on work published in the last 20 years. This reflects the clearly perceived need for a continued and increased understanding of mite behaviour and biology, and a recognition of the importance of the damaging and beneficial effects that mites have on human activities. The ecological and behavioural diversity of mites is also reflected in the methods used to collect and study them. Methods used to collect mites are described in a straightforward practical way, as are the methods used for preparing specimens for microscopic study. The authors correctly point out that important advances have been made in electron microscopy and electronic imaging, and these technologies can only become more important in the future, as tools for both research and illustrating.

The introductory section is followed by a deceptively short chapter on mite classification. The authors have used the results of recent cladistic analyses to identify monophyletic higher taxa of mites, using evidence from both molecular data and sophisticated comparative morphology. The resulting classification is the outcome of a lively discussion of the higher classification of mites that has been going on for the last 20 years or more. Some of the results have been foreshadowed elsewhere. The mites are provisionally considered to be a single monophyletic taxon, the Subclass Acari. The Astigmata are now recognised as a derivative group of oribatids, placed within the oribatid Supercohort Desmonomatides. This decision has some effects that are rather alarming at first. On page 38, the illustrations of "Some oribatid body types" include representatives of the Chaetodactylidae and Rhyncoptidae, and it's hard to think of these families as oribatids. References to a familiar genus of Astigmata like Sancassania as "Oribatida, Acaridae" will take some getting used to. The group of families previously known as Endeostigmata has long been recognised as heterogeneous, but they have normally been grouped together within the Prostigmata (or the same group under the alternative name Actinedida). The potential breakup of this group was suggested in the 1978 Manual and elsewhere, but is now formalised. Most of the families of Endeostigmata are now transferred to the Order Sarcoptiformes, and only the Lordalycidae and Sphaerolichidae remain in the Order Trombidiformes, in a Suborder Sphaerolichida, the sister group of the Suborder Prostigmata. The taxonomic ranking of various other taxa is either raised (for example, the Sejina become a Suborder) or lowered (the Parasitengona are placed within the Supercohort Anystides). The resulting classification is sometimes awkward and inconsistent. Some Suborders are divided into Cohorts, others into Supercohorts or directly into Superfamilies. This is a compromise solution, forced by the enormous variation in size and diversity of some of the subordinate taxa. The authors also pragmatically retain the unorthodox use of the category Cohort between Order and Superfamily levels, instead of at a much higher level as in the rest of the Animal Kingdom. These changes mean it was necessary to prepare a completely new key to mite Superorders, Orders, and Suborders. They also require no fewer than 52 explanatory notes, to explain how the higher taxa used here are related to those used in previous classifications. It was unnecessary and probably futile to introduce new standardised suffixes for the names of higher taxa. Eupodides and Dermanyssiae are unlikely to catch on in common usage as substitutes for Eupodina and Dermanyssina. The authors freely acknowledge that the higher classification presented here is not the final word, but will continue to evolve and change as basic taxonomic knowledge and technology both improve. The fact that over 100 new families of mites have been described since the previous edition is clear evidence that the classification of the mites is still extremely fluid. One of the major areas of instability is still the Parasitengona (now re-badged as Cohort Parasitengonina). The text on this group, and the key to families of water mites, include a passionate plea for more rearing studies to clarify the relationships between larval and adult taxa, especially from the Southern Hemisphere. This is just one of many 
areas where a greatly increased and sustained taxonomic effort is desperately needed.

Most of the book is dedicated to a systematic march through the Orders and Suborders of mites. Each section includes a totally new key to families, diagnoses of superfamilies, and a summary of the key taxonomic features of each family. The biological information about each family is now much more comprehensive, with data on ecology, host relationships, life cycles, and economic significance where it is available. These family-level essays will be the essential starting point for the study of the taxonomy and biology of any mite group for a whole new generation of research projects. Here too, the authors of individual chapters have used cladistic methods to produce a revised classification based on hypothetical phylogenetic relationships. And here too, the results include some unfamiliar combinations of taxa. The genera that were included in the family Ascidae are now distributed over three families in two superfamilies. The chapter on ticks includes a new key to genera, incorporating the synonymy of Aponomma with Amblyomma, and the relegation of Boophilus to a subgenus of Rhipicephalus. The group that was known as the Anystina has undergone a dramatic change of personality, losing the Pterygosomatidae and gaining four families that were previously widely scattered in different Cohorts. The section on Oribatids (excluding Astigmata) is greatly expanded and completely reorganised, and features a great number of new illustrations. The Astigmata, now referred to as Cohort Astigmatina, are formally recognised as a subgroup of oribatids, but still qualify for a separate chapter. The close relationship between these groups is emphasised by the application of some oribatid terminology to replace some familiar names used for structures in the Astigmata. The great growth in knowledge of these mites in recent years is reflected in the extensive and completely new reviews of their taxonomy and biology that are presented here, both in general and at the family level. The result is a very welcome synthesis of a scattered and disparate literature, combined with the author's deep personal knowledge of the group. The "Psoroptidea" is recognised provisionally as a monophyletic group, but is not named as a taxon or allocated to any particular rank, presumably because this would require complicated and undesirable changes to the ranking and names of the non-psoroptid superfamilies.

The technical production quality of this new edition is outstanding. The binding and paper are of the best international quality, and the text is attractive and easy to read. Many of the illustrations are familiar, but have survived the recycling process remarkably well. These are supplemented by hundreds of new illustrations, and all the illustrations have new labels that reflect an increasingly sophisticated understanding of mite anatomy and morphology. The editors have made a big investment in time and effort to obtain permission to reprint illustrations that were originally published elsewhere. Some of these illustrations are beautiful portraits of whole mites, which a modern generation of acarologists would do well to emulate, if they have the necessary skills. The scanning electron microscope photos are very dramatic, and serve as a preview of the more extensive use of this medium in the future. The new comprehensive subject index is a very welcome complement to the taxonomic index. A further big improvement is the consolidation of all the cited literature into a single enormous bibliography of more than 4,000 references. It is slightly frustrating to find that the illustrations are still grouped into blocks at the end of each chapter. This sometimes means that cited illustrations are many pages away from the associated text, which causes a lot of unnecessary page-turning.

The editors have done a fine job of weaving together the separate chapters contributed by different authors. The feel and style of the text flows smoothly from one chapter to another, and cross-references between chapters work well. It will be easy to forget that citations of this work should be to the authors of individual chapters, not to Krantz and Walter (2009) as a whole. It is inevitable in a work of this monumental size and complexity that there will be occasional typographical errors and minor slips, but I saw remarkably few. The publisher's advertised price of USD $\$ 175.00$ is very reasonable for a book of this size and importance. 
The Third Edition of the Manual is a superb review of the science of acarology to the end of 2006. It will be the fundamental textbook of acarology for the next 30 years, as the previous edition was for the last 30 years. Its comprehensive review of the systematics and biology of mites should inspire a whole new generation of young scientists to take up the study of acarology, as well as serving as the basic reference for established researchers and teachers in acarology, entomology, and the plant and animal sciences.

Accepted by Z.-Q. Zhang: 27 May 2009 